\title{
Spatial correlation functions and dynamical exponents in very large samples of four-dimensional spin glasses
}

\author{
Lucas Nicolao, ${ }^{1, *}$ Giorgio Parisi, ${ }^{2}$ and Federico Ricci-Tersenghi ${ }^{2}$ \\ ${ }^{1}$ Dipartimento di Fisica, Sapienza Università di Roma, P.le Aldo Moro 2, I-00185 Roma, Italy \\ ${ }^{2}$ Dipartimento di Fisica, Istituto Nazionale di Fisica Nucleare-Sezione di Roma I, Istituto per i Processi Chimico-Fisici, \\ Consiglio Nazionale delle Ricerche-Unità Organizzativa di Supporto Roma, Sapienza Università di Roma, \\ P.le Aldo Moro 2, I-00185 Roma, Italy
}

(Received 9 January 2014; published 24 March 2014)

\begin{abstract}
The study of the low temperature phase of spin glass models by means of Monte Carlo simulations is a challenging task, because of the very slow dynamics and the severe finite-size effects they show. By exploiting at the best the capabilities of standard modern CPUs (especially the streaming single instruction, multiple data extensions), we have been able to simulate the four-dimensional Edwards-Anderson model with Gaussian couplings up to sizes $L=70$ and for times long enough to accurately measure the asymptotic behavior. By quenching systems of different sizes to the critical temperature and to temperatures in the whole low temperature phase, we have been able to identify the regime where finite-size effects are negligible: $\xi(t) \lesssim L / 7$. Our estimates for the dynamical exponent $(z \simeq 1 / T$ ) and for the replicon exponent ( $\alpha \simeq 1.0$ and $T$ independent), that controls the decay of the spatial correlation in the zero overlap sector, are consistent with the replica symmetry breaking theory, but the latter differs from the theoretically conjectured value.
\end{abstract}

DOI: 10.1103/PhysRevE.89.032127

PACS number(s): 75.10.Hk, 75.50.Lk, 75.40.Mg

\section{INTRODUCTION}

Even though much progress has been made in the past decades, our comprehension of the underlying nature of the spin glass phase in finite dimensions faces many open problems [1]. The two major scenarios stem from theories that are exact in opposite dimensional limits. Exact in one dimension, the droplet picture [2,3] considers only two equilibrium pure states related by spin-flip symmetry. In contrast, in the mean-field picture [4], replica symmetry is fully broken in a hierarchical pattern and the many equilibrium pure states are organized in an ultrametric fashion $[5,6]$.

These central features of the mean-field solution survive in finite dimensions, as the mean-field solution is amenable to computations down to, and around, the upper critical dimension $\left(d_{u}=6\right)$, in the form of a replicated field theory. It is well known that at and below the critical temperature there is a massless mode associated with the breaking of the continuous replica symmetry. Therefore, a spin glass is always in a critical state due to the coexistence of many equilibrium states, and the associated overlap-overlap connected correlation functions decay as a power law. This Goldstone mode is called the replicon mode. In this work we will present results restricted to the zero overlap sector. ${ }^{1}$ Note that since there is no replica symmetry breaking in the droplet theory (only two pure states exist) the overlap-overlap correlation function is not even defined for the zero overlap sector, and in the $q_{\mathrm{EA}}$ sector, where it is defined, it decays algebraically with the standard droplet scaling exponent $\theta$.

\footnotetext{
*Present address: Departamento de Física, Universidade Federal do Rio Grande do Sul CP 15051, 91501-979, Porto Alegre, Brazil.

${ }^{1}$ Correlations are expected to behave differently in other overlap sectors. Results for the replicon exponent for different overlap sectors will appear in a forthcoming publication.
}

As it happens in the wider framework of the renormalization group for random systems, spin glasses in zero magnetic field face technical difficulties, especially for long distance behavior in $6-\epsilon$ dimensions inside the broken phase [7]. At this point, numerical simulations are very useful and we feel that studying the four-dimensional case is very important to interpolate between the field theoretical results above $d_{u}$ and the tridimensional case. The latter is the most explored case in large scale numerical simulations, which is indeed the crucial case, but also very close to the lower critical dimension (most probably $d_{l} \simeq 2.5[8,9]$ ).

Above $d_{u}=6$, the strongest infrared behavior among all propagators is exhibited by the zero overlap replicon, where the associated overlap-overlap correlations decay as $r^{-\alpha}$ with $\alpha=$ $d-4$ below $T<T_{c}$ and $\alpha=d-2$ at $T=T_{c}$ [10]—verified by numerical simulations in large- $d$ diluted hypercubes [11] and in $d=6$ [12]. This scenario should change for $d<6$, leading to the standard relation $\alpha=d-2+\eta$ at $T=T_{c}$ ( $\eta$ being the anomalous dimension). Moreover field theoretical arguments suggest [13] that $\alpha=(d-2+\eta) / 2$ for $T<T_{c}$.

The large majority of works devoted to the numerical estimation of the replicon exponents have been done by Monte Carlo simulations of tridimensional models. Nonequilibrium methods [14-17] are an alternative to equilibrium studies [18-20], presenting compatible values; the latest estimates are $\alpha \simeq 0.4$ at $T<T_{c}$ for the zero overlap sector.

Nonequilibrium methods rely on the extrapolation of the dynamical evolution to allow one to estimate the equilibrium correlation functions [15], assuming a simple and yet general ansatz [14] for the time dependence in the overlap-overlap correlation function. However, a very powerful method based on a set of integral estimators of characteristic length scales was introduced recently [17], allowing a more robust and ansatz-independent determination of the equilibrium correlation functions. Although such nonequilibrium methods allow the study of equilibrium spatial correlations only in a 
restricted overlap sector, they benefit from the use of very large lattices and thus having finite-size effects under control, while equilibration of large system sizes deep in the cold phase is computationally cumbersome.

The only previous numerical determination of the fourdimensional case used a similar nonequilibrium analysis based on the definition of an ansatz, but in rather small system sizes and time windows $[L \leqslant 26$ and $t<60000$ Monte Carlo (MC) steps], with a replicon exponent lying in the range $0.9<\alpha<1.35$ below $T_{c}$ [21]. In this work we report, using nonequilibrium methods mentioned above, an almost constant in temperature replicon exponent $\alpha \simeq 1.0$ and the dynamical critical exponent (inversely proportional to $T$ ) with high accuracy, using unprecedented sizes and time range, where we can observe clearly the finite-size effects and have them under control.

\section{MODEL AND THE CORRELATION FUNCTION}

We have simulated the Edwards-Anderson model for spin glasses on a four-dimensional cubic lattice of volume $L^{4}$ with helicoidal boundary conditions. The Hamiltonian is

$$
\mathcal{H}=-\sum_{\langle\mathbf{x}, \mathbf{y}\rangle} J_{\mathbf{x y}} \sigma_{\mathbf{x}} \sigma_{\mathbf{y}}
$$

where $\sigma_{\mathbf{x}}= \pm 1$ are Ising spin variables located at lattice position $\mathbf{x}$ and $J_{\mathbf{x y}}$ are quenched coupling constants joining pairs of lattice nearest neighbors (denoted by $\langle\mathbf{x}, \mathbf{y}\rangle$ ), drawn from a Gaussian probability distribution of zero mean and unitary variance.

Our study concentrates on the behavior of the correlations of the replica field $q_{\mathbf{x}}(t)=\sigma_{\mathbf{x}}^{(1)}(t) \sigma_{\mathbf{x}}^{(2)}(t)$ :

$$
C_{4}(\mathbf{r}, t)=\overline{L^{-4} \sum_{\mathbf{x}} q_{\mathbf{x}}(t) q_{\mathbf{x}+\mathbf{r}}(t),}
$$

where $\sigma^{(1)}$ and $\sigma^{(2)}$ are two real replicas, meaning two independent systems evolving with the same couplings. We denote by $\overline{(\cdots)}$ the average over different realizations of disorder. In our study we have used the data for $C_{4}(\mathbf{r}, t)$ measured along the directions of the principal axis. We have not found significant improvement of the statistical errors by averaging $C_{4}(\mathbf{r}, t)$ over spherical shells.

We always consider the time evolution of this system quenched from high temperatures (initial conditions are chosen randomly, i.e., $T=\infty$ ) to a fixed working temperature, below or at the estimated critical temperature $T_{c}=$ 1.805(10) [22]. To mimic the physical evolution we have used the standard Metropolis dynamics. Since we start with two uncorrelated replicas, they will typically relax in two orthogonal valleys, so that the system will remain in the $q=0$ sector for large times (much longer than times used in this study, due to the very large lattices we simulate). As a consequence, from dynamics we extract the properties of the equilibrium $q-q$ correlation in the zero overlap sector.

In order to reach large-scale space and time regimes, we have developed an optimized code dedicated to the use of single instruction, multiple data (SIMD) technology, present in practically every modern CPU, where a single processor is able to perform four floating-point operations simultaneously. With
TABLE I. Set of the simulation parameters. Configurations of the two replicas at each $2^{i / 2} \mathrm{MC}$ step where saved to disk, as well as the couplings realization.

\begin{tabular}{lccc}
\hline \hline$T$ & $L$ & $N_{S}$ & MC steps \\
\hline 1.805 & 70 & 40 & $2^{22.5}$ \\
& 42 & 20 & $2^{23}$ \\
1.400 & 30 & 90 & $2^{23}$ \\
& 70 & 34 & $2^{19.5}$ \\
& 42 & 20 & $2^{23}$ \\
1.263 & 30 & 60 & $2^{23}$ \\
& 70 & 50 & $2^{21}$ \\
& 54 & 20 & $2^{21.5}$ \\
1.100 & 42 & 36 & $2^{26}$ \\
& 30 & 211 & $2^{23}$ \\
& 54 & 38 & $2^{21}$ \\
0.900 & 42 & 16 & $2^{22}$ \\
& 30 & 60 & $2^{23}$ \\
0.700 & 54 & 40 & $2^{20}$ \\
0.540 & 30 & 91 & $2^{23}$ \\
\hline \hline
\end{tabular}

the help of streaming SIMD extensions (SSE) instructions [23] we could benefit from this intrinsic parallelization to perform all the operations involved in a Monte Carlo simulation (see Ref. [24] for the linear congruential pseudorandom number generator and Ref. [25] for the implementation of the exponential function), updating four noninteracting spins simultaneously, one in each quarter of the whole volume.

Since this optimization pushes the processor performance to its theoretical limit, the overall computation time is strongly affected by the type and size of the cache memory, as well as its availability. For an Intel Xeon CPU X5365 at $3.00 \mathrm{GHz}$ with a L2 Cache of $4 \mathrm{MB}$, the speedup is 19 times faster than an equivalently optimized but nonvectorized code.

We have simulated the off-equilibrium dynamics using linear sizes ranging from $L=30$ to 70 , for seven temperatures ranging approximately from $0.3 T_{c}$ to $0.8 T_{c}$, plus $T_{c}$ - see the complete set of simulation parameters in Table I. For each sample, we saved to disk the couplings and the configurations of each replica at $2^{i / 2} \mathrm{MC}$ steps; the analysis was performed offline.

\section{RESULTS}

First we analyze the overlap correlation function starting with an ansatz known to be a good representation of its functional form [14]:

$$
C_{4}(r, t)=\frac{\text { const }}{r^{\alpha}} \exp \left\{-[r / \xi(t)]^{\delta}\right\}
$$

Assuming that the coherence length grows algebraically as $\xi(t)=B t^{1 / z}$, and following Ref. [15], we perform a fit to Eq. (3) in two steps. First the time dependence for each fixed distance is fitted to

$$
-\ln C_{4}(r, t)=A(r)+B(r) t^{-\delta / z},
$$


TABLE II. Best fitting parameters and corresponding fitting ranges obtained by interpolating spatial correlation functions with the ansatz in Eq. (3). In the cases marked by * a second order term, $t^{-2 \delta / z}$, has been added to Eq. (4).

\begin{tabular}{lccccccc}
\hline \hline$T$ & $L$ & {$\left[r_{i}, r_{f}\right]$} & $\delta$ & $B^{-\delta}$ & $z$ & {$\left[r_{i}, r_{f}\right]$} & $\alpha$ \\
\hline 1.400 & 70 & {$[3,11]$} & $1.47(4)$ & $1.00(5)$ & $6.18(32)$ & {$[1,7]$} & $1.10(6)$ \\
& 42 & {$[3,8]$} & $1.49(5)$ & $0.98(9)$ & $6.26(48)$ & {$[1,8]$} & $1.11(4)^{*}$ \\
1.263 & 70 & {$[3,12]$} & $1.48(3)$ & $0.96(2)$ & $6.79(17)$ & {$[1,5]$} & $1.07(2)^{*}$ \\
& 42 & {$[3,12]$} & $1.52(8)$ & $0.78(2)$ & $7.68(37)$ & {$[1,6]$} & $1.05(3)^{*}$ \\
& 30 & {$[3,10]$} & $1.48(4)$ & $0.92(4)$ & $6.91(37)$ & {$[1,6]$} & $1.09(2)^{*}$ \\
1.100 & 54 & {$[3,9]$} & $1.51(4)$ & $0.88(6)$ & $7.82(74)$ & {$[1,4]$} & $1.04(6)$ \\
& 30 & {$[3,9]$} & $1.52(8)$ & $0.76(6)$ & $8.9(1.5)$ & {$[1,7]$} & $1.03(7)^{*}$ \\
0.900 & 30 & {$[3,8]$} & $1.51(7)$ & $0.77(6)$ & $10.1(2.2)$ & {$[1,4]$} & $1.05(7)^{*}$ \\
0.700 & 30 & {$[2,6]$} & $1.50(4)$ & $0.86(4)$ & $11.0(1.6)$ & {$[1,3]$} & $1.08(11)$ \\
0.540 & 30 & {$[2,6]$} & $1.54(6)$ & $0.81(3)$ & $13.0(2.8)$ & {$[1,3]$} & $1.09(15)$ \\
\hline \hline
\end{tabular}

from which the optimal $\delta / z$ is determined through the minimization of a spatially averaged $\chi^{2}$,

$$
\hat{\chi}^{2}=\frac{1}{r_{\mathrm{M}}-2} \sum_{r=3}^{r_{\mathrm{M}}} \chi^{2}(r) / N_{\mathrm{DOF}}(r),
$$

up to distance $r_{\mathrm{M}}$ where fit to Eq. (4) is still meaningful. Then we interpolate the coefficients in Eq. (4) with the laws $B(r)=$ $B^{-\delta} r^{\delta}$ and $A(r)=$ const $+\alpha \ln (r)$ at the optimal $\delta / z$ to obtain the best estimates for the exponents $\alpha, \delta$, and $z$. In general the $\hat{\chi}^{2}$ minimization has been performed for distances $r \geqslant 3$. However, in some cases we found it necessary to use shorter distances for the estimate of $\alpha$ and eventually a quadratic term $C(r) t^{-2 \delta / z}$ in Eq. (4) has been added.

Table II summarizes the best estimates obtained with this procedure. With high accuracy the exponent of the stretched exponential is constant throughout the low temperature phase with value $\delta=1.50(1)$. The dynamical critical exponent dependence on the temperature is very well described by the law $z \simeq 8.9(2) / T$ and the replicon exponent is nearly constant with average $\alpha \simeq 1.06(6)$.

This fitting method provides a reliable estimation for the exponents and it is certainly a robust way to get the $t \rightarrow \infty$ limit through a global fit [21] to the ansatz in Eq. (3). Still it suffers from some drawbacks: it is ansatz dependent and some technical aspects are not perfectly under control. For example, in order to ensure a fair spatial average of the $\hat{\chi}^{2}$ in the first step of the procedure, short distances have to be carefully selected in order not to dominate over the longer distances. It can be difficult to determine precisely whether this time window sits between an initial fast transient dynamics and a near equilibrium dynamics when $\xi(t) \gg r$.

To overcome these issues, we move to an ansatzindependent method to estimate $z$ and $\alpha$, through a set of integrals of the correlation function in the form

$$
I_{k}(t) \equiv \int_{0}^{L / 2} d r r^{k} C_{4}(r, t),
$$

and, since we expect a scaling form $C_{4}(\mathbf{r}, t) \sim r^{-\alpha} f\left(\frac{r}{\xi}\right)$, then

$$
I_{k}(t) \propto \xi(t)^{k+1-\alpha},
$$

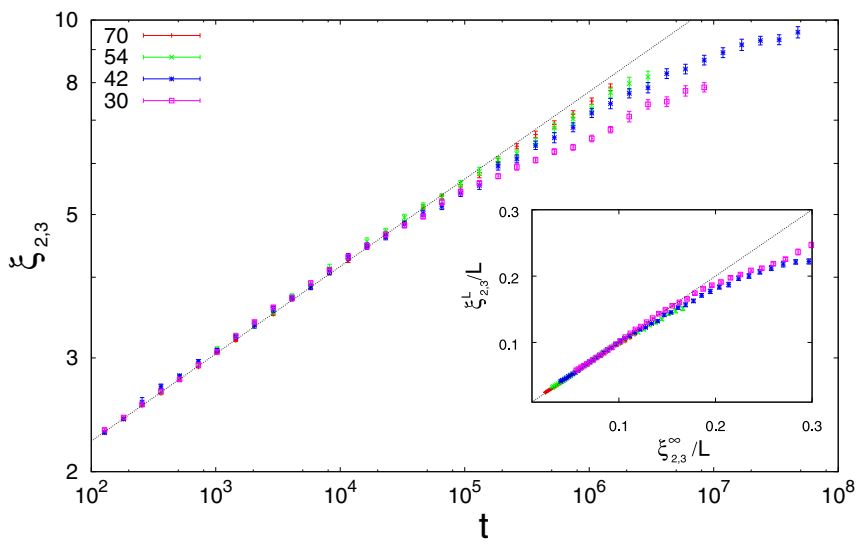

FIG. 1. (Color online) Time dependence of the coherence length for $T=1.263$ and all system sizes simulated. The line accounts for the power-law fit to $L=70$ data in the range $\xi_{2,3} \in[3,5]$, that corresponds to $\xi_{2,3}^{\infty}(t) \equiv 1.20(1) t^{1 / 7.44(10)}$. Inset: The scaling function of the coherence length, evidencing the point where finite-size effects become important, $L \lesssim 6.5 \xi_{2,3}(t)$.

and we can estimate the coherence length as

$$
\xi_{k, k+1}(t) \equiv \frac{I_{k+1}(t)}{I_{k}(t)} .
$$

From Eqs. (7) and (8) it is possible to estimate the replicon exponent $\alpha$. For example, in case the correlation function is given exactly by Eq. (3) with $\alpha=1$ and $\delta=1.5$ we have that

$$
\xi_{2,3}(t)=\xi(t) / \Gamma(4 / 3)=1.12 \xi(t),
$$

with the constant weakly depending on $\alpha$ and $\delta$.

In order to evaluate the integrals above, we adopt the same procedure used in Refs. [17,26], introducing a self-consistent integration cutoff at a distance where the correlation function first becomes less than $X$ times its statistical error, with $X=5$ but for $T=T_{c}(X=7)$ and $T=0.9(X=4)$. As this method alleviates the integrals from the wide fluctuations of the nonself-averaging tails, it also induces a systematic error. To avoid such a systematic error, we estimate the contribution of the tail by performing a fit to the ansatz in Eq. (3) using our previous estimates of $\delta \simeq 1.5$ and $\alpha \simeq 1.0$ for $T<T_{c}$ and the best previous estimate $[22,27] \alpha \simeq 1.7$ for $T=T_{c}$. The fit is performed in the range $\left[3, \min \left(r_{\max }, r_{\text {cutoff }}\right)\right]$ with $r_{\max }=10$, 12 , and 15 for $L=30,42$, and $L \geqslant 54$ respectively, and is used to estimate the integral in the range $\left[r_{\text {cutoff }}, L / 2\right]$.

In Fig. 1 we report the time dependence of $\xi_{2,3}(t)$ for various system sizes at $T=1.263$. In the early stage of the dynamics, in general for $\xi(t) \lesssim 3$, spatial correlation functions show the effects due to the lattice discretization and the growth of $\xi(t)$ shows some preasymptotic behavior. In the inset of Fig. 1 we show that finite-size effects come into play when $L \lesssim 6.5 \xi_{2,3}(t) \simeq 7 \xi(t)$, much earlier than the standard expectation, $\xi(t) \simeq L / 2$. In addition to the finite-size effects, we also observe some deviations due to the uncertainty in estimating the contribution of the tail. As a consequence the estimation of the $z$ exponent from the fit $\xi_{2,3}(t) \propto t^{1 / z}$ must be restricted to a time window that excludes both the short time dynamics (affected by lattice discretization) and the very long time dynamics, even for the largest volumes. 


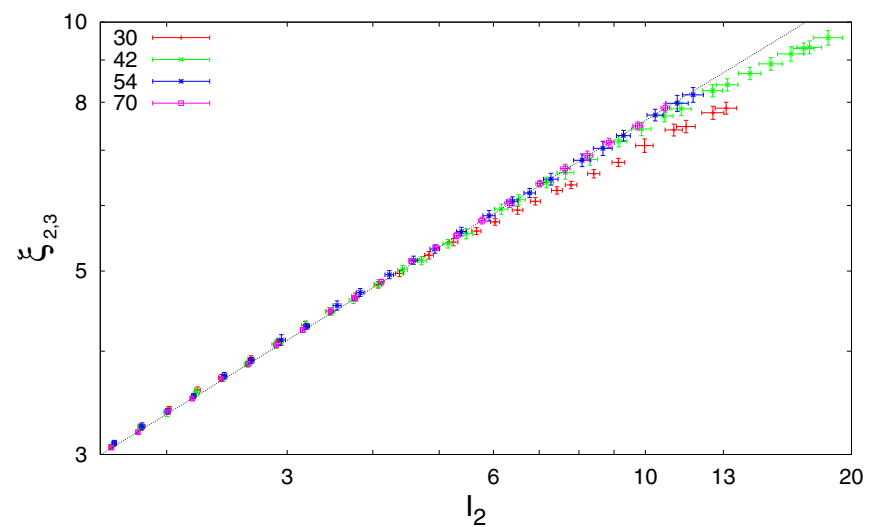

FIG. 2. (Color online) Data for $T=1.263$; different sizes have different numbers of data points, according to the length of the simulation shown in Table I. The line corresponds to $\alpha=1.02$ obtained from fitting the $L=70$ data. Deviations from this line are finite-size effects. Although the simulations for $L=42$ have been run longer for a factor 32, it is clear that the last part of the simulation is affected by strong finite-size effects and practically useless for the estimation of the exponent.

Fortunately enough, all integrals $I_{k}$ experience the same inaccuracy in the extrapolation of the tail contribution, so that these errors compensate each other in the relation between $\xi_{k, k+1}$ and $I_{k}$. In Fig. 2 we clearly identify the finite-size effects, but there are no other systematic errors due to the tail extrapolation. Since $I_{2} \propto \xi_{k, k+1}^{3-\alpha}$ we can extract the replicon exponent from a direct fit to the relation Eq. (7), without discarding late time data for the largest sizes. We use a standard method for linear fits with errors in both coordinates [28].

In principle, we could have used other values for $k$ and $m$ in $\xi_{k, k+1}$ and $I_{m}$ appearing in the relation Eq. (7). The choice for $\xi_{2,3}$ and $I_{2}$ is justified because it brings the highest amount of points for the fits of $\xi_{k, k+1}\left(I_{k}\right)$ to a power law, namely, from 20 to $50 \%$ less discarded short time data. To keep consistency with the $\alpha$ estimation, we have chosen $\xi_{2,3}$ for the estimate of exponent $z$.

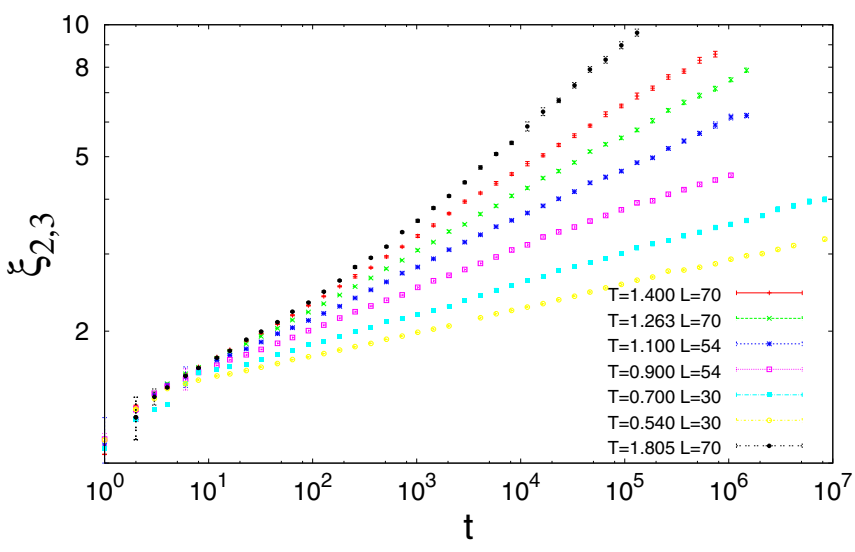

FIG. 3. (Color online) Time dependence of the coherence length $\xi_{2,3}$ for the largest size $L=70$ and several temperatures, ranging from the critical one $T_{c}=1.805$ down to very low temperatures.

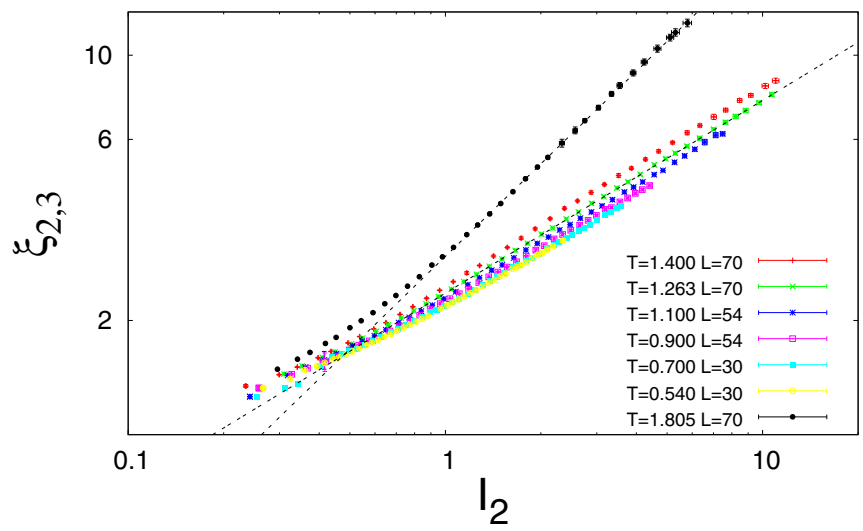

FIG. 4. (Color online) Data for $L=70$ and several temperatures. The relation $\xi_{2,3} \propto I_{2}^{1 /(3-\alpha)}$ provides, at large times, a reliable estimate for the replicon exponent $\alpha$. Lines correspond to $\alpha=$ 1.02 for $T=1.263$ and $\alpha=1.77$ for $T=T_{c}=1.805$. The latter exponent corresponds to $\eta=-0.23(1)$, which is compatible with the latest estimate using equilibrium finite-size scaling analysis [22]: $\eta=-0.275(25)$.

Our main results are summarized in Figs. 3 and 4, where $\xi_{2,3}(t)$ is shown as a function of time and $I_{2}(t)$, respectively, for several temperatures. From data in Fig. 3 we estimate the dynamical exponent $z$ by fitting to a power law in the range $\xi \in\left[\xi_{\min }, \xi_{\max }\right]$, and from the data in Fig. 4 we get the replicon exponent $\alpha$ fitting in the range $\xi \geqslant \xi_{\min }$. It is immediately clear from the observation that all data with $T<T_{c}$ in Fig. 4 become parallel at large times that $\alpha$ is roughly constant in the low temperature phase. Our best estimates for the exponents $z$ and $\alpha$ are reported in Table III, together with the fitting ranges, the corresponding $\chi^{2}$, and the number of degrees of freedom $\left(N_{\mathrm{DOF}}\right)$. In Fig. 5 we plot the best estimate for the exponents $z$ and $\alpha$ in a way that makes evident that

TABLE III. Best estimates for the dynamical and replicon exponents from the fitting of the data shown in Figs. 3 and 4. The fits are performed on the range $\left[\xi_{\min }, \xi_{\max }\right]$ for $z$, and with $\xi_{2,3} \geqslant \xi_{\min }$ in the case of $\alpha$. Errors are obtained through jackknife methods.

\begin{tabular}{llcccc}
\hline \hline$T$ & {$\left[\xi_{\min }, \xi_{\max }\right]$} & $z$ & $\chi_{\xi}^{2} / N_{\text {DOF }}$ & $\alpha$ & $\chi_{I_{2}(\xi)}^{2} / N_{\text {DOF }}$ \\
\hline 1.805 & {$[3.5,13.0]$} & $4.95(04)$ & $19 / 21$ & $1.766(03)$ & $3.4 / 21$ \\
1.400 & {$[3.5,8.0]$} & $6.86(14)$ & $5.1 / 16$ & $1.055(19)$ & $1.5 / 18$ \\
& {$[4.0,8.0]$} & $6.89(17)$ & $3.3 / 14$ & $1.050(22)$ & $0.7 / 16$ \\
1.263 & {$[3.0,5.0]$} & $7.44(10)$ & $6.2 / 11$ & $1.020(10)$ & $3.4 / 20$ \\
& {$[3.0,5.5]$} & $7.45(08)$ & $7.1 / 13$ & & \\
& {$[3.5,5.5]$} & $7.46(10)$ & $3.4 / 10$ & $1.015(13)$ & $2.1 / 17$ \\
1.100 & {$[3.0,5.5]$} & $9.15(15)$ & $4.0 / 15$ & $0.996(19)$ & $7.8 / 20$ \\
& {$[3.5,5.5]$} & $9.21(20)$ & $3.4 / 11$ & $1.024(29)$ & $5.5 / 16$ \\
& {$[3.0,6.5]$} & $9.19(16)$ & $7.8 / 20$ & & \\
0.900 & {$[3.5,6.5]$} & $9.23(27)$ & $6.6 / 16$ & & \\
& {$[2.7,4.0]$} & $11.32(33)$ & $6.6 / 14$ & $0.909(31)$ & $1.8 / 19$ \\
0.700 & {$[3.0,4.0]$} & $11.50(46)$ & $5.9 / 10$ & $0.921(42)$ & $1.4 / 15$ \\
& {$[3.7,4.5]$} & $15.31(67)$ & $2.3 / 18$ & $0.900(36)$ & $1.1 / 18$ \\
0.540 & {$[2.3,4.5]$} & $15.47(77)$ & $1.3 / 14$ & $0.923(40)$ & $0.4 / 14$ \\
& {$[2.6,3.3]$} & $17.9(1.3)$ & $12 / 17$ & $0.896(39)$ & $2.0 / 17$ \\
\hline \hline
\end{tabular}




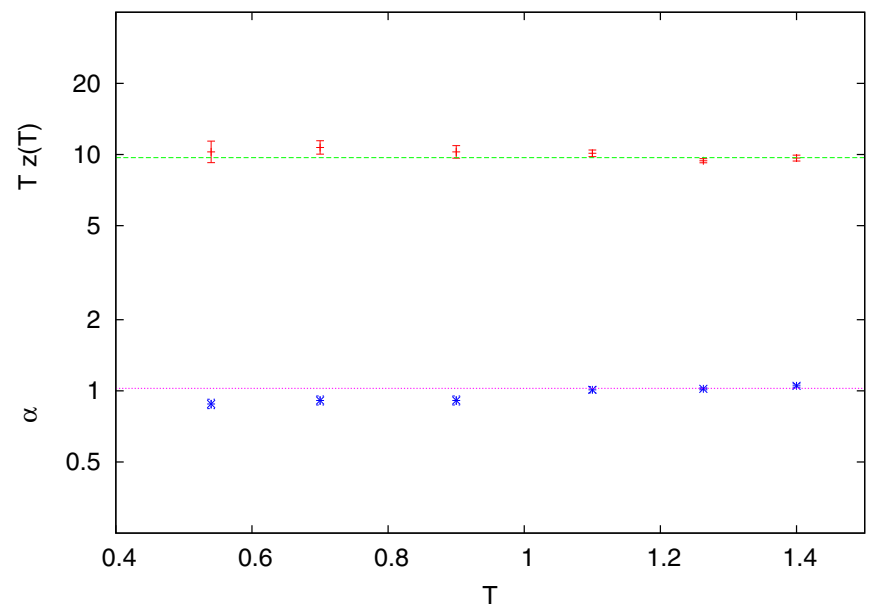

FIG. 5. (Color online) Best estimates for $z(T)$ multiplied by $T$ (above) and $\alpha$ (below) taken from Table III. Lines are best fits: $T z(T)=9.7(2)$ and $\alpha=1.025(9)$.

$z(T) \simeq 9.7(2) / T$ and $\alpha \simeq 1.025(9)$ for $T<T_{c}$. Actually the best value for $\alpha$ has been estimated only from data in the range $T \in[1,1.5]$, because for lower temperatures we observe a systematic decrease in the $\alpha$ value that we explain as follows. From data plotted in Fig. 4 we see that systems at the lowest temperatures are still approaching the asymptotic dynamics; so, it is likely that the small drift of the exponents for these low temperatures does not reflect a real change, but rather a preasymptotic effect due to a not large enough value of $\xi(t)$. Moreover in the $T=0$ limit we could have in principle a different exponent and we are maybe observing the beginning of the crossover region. Please note that the relatively small sizes used at the lowest temperatures (see Table I) do not induce any finite size effect, because the growth of $\xi(t)$ is extremely slow and barely reaches $\xi \sim 4<L / 7$.

\section{CONCLUSIONS}

We have performed an extensive numerical study of the four-dimensional Gaussian Edwards-Anderson model with the

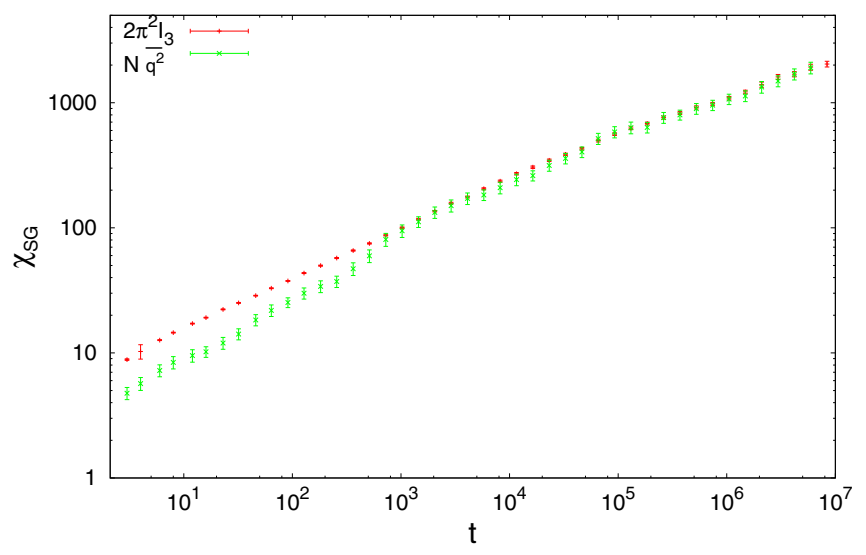

FIG. 6. (Color online) In order to check the reliability of the integral estimator $I_{3}$, we compare it to the SG susceptibility for the $L=30$ and $T=1.263$ case, where we have the largest number of samples (211). aim of measuring the dynamical and the replicon exponents. We have used very large system sizes (up to $70^{4}$ ), which were never used before. These huge sizes are required to overcome finite-size effects, which appear when the coherence length $\xi(t)$ is of the order of $1 / 7$ of the system size. The values of the replicon exponent $\alpha$, controlling the spatial decay of the correlation function, $C_{4}(r, t) \sim r^{-\alpha}$, are roughly constant in the low temperature region (apart from some preasymptotic effects at very low temperatures). Our final conservative estimate is $\alpha=1.03(2)$, which is not far but definitely different from the one conjectured by the field theoretical arguments based on the analysis of the first order in the $\epsilon=6-d$ expansion, $(d-2+\eta) / 2=0.883$. We also confirm that the dynamical exponent $z(T)$ is inversely proportional to the temperature in the entire temperature range we studied.

\section{ACKNOWLEDGMENTS}

L. Nicolao would like to thank David Yllanes for the enlightening discussions. The authors acknowledge financial support from the European Research Council (Grant No. 247328) and from the Italian Research Ministry (Fondo per gli Investimenti della Ricerca di Base Project No. RBFR086NN1). L. Nicolao also acknowledges the initial financial support from Conselho Nacional de Desenvolvimento Científico e Tecnológico, Brazil.

\section{APPENDIX: A CLOSER LOOK AT INTEGRAL ESTIMATORS}

In this Appendix we explore in some detail the properties of the integrals $I_{k}$ defined by Eq. (6) as well as the related estimators for the coherence length defined by Eq. (8). Note that the spin glass susceptibility is given by $\chi^{\mathrm{SG}}(t)=$ $2 \pi^{2} I_{3}(t)$. This relation offers a check for the correctness of the computation of the integrals. Since $\chi^{\mathrm{SG}}=N \overline{q^{2}}$ is a

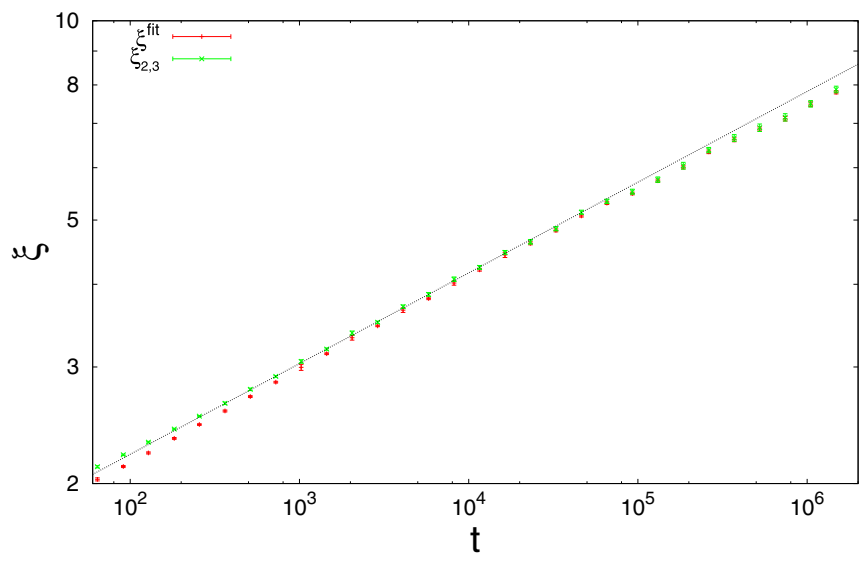

FIG. 7. (Color online) Comparison between the integral estimator for the coherence length, $\xi_{2,3}$, and the $\xi^{\text {fit }}$ obtained by fitting to the ansatz in Eq. (3). Data are for $L=70$ and $T=1.263$ and the values of $\xi^{\text {fit }}$ have been multiplied by a factor $1 / \Gamma(4 / 3)=1.12$ in order to be equal to $\xi_{2,3}$ in case the ansatz in Eq. (3) is asymptotically exact. Fitting these data in the range $3<\xi<5$ we get $z\left(\xi_{2,3}\right)=7.4(1)$ and $z\left(\xi^{\mathrm{fit}}\right)=7.23(7)$. We draw a line of slope $1 / 7.3$ as a guide for the eye. 
non-self-averaging quantity we do such a comparison for the case in which we dispose the largest set of samples (see Fig. 6).

A comparison between the integral estimator $\xi_{2,3}$ for the coherence length and another estimator $\xi^{\text {fit }}$ can be seen in Fig. 7. The latter is obtained by fitting correlation functions $C_{4}(r, t)$ with the ansatz in Eq. (3) with $\alpha=1.0$ and $\delta=1.5$.
These two estimators are in agreement with each other, once normalized according to Eq. (9). A closer inspection reveals the deviation of $\xi_{2,3}$ at larger times due to the badness of the estimation of the tail contribution, so that a secure range for a fit to obtain $z$ is $\xi_{2,3} \in[3,5]$, though compatible results are obtained in a wider time window, as can be seen in Table III.
[1] G. Parisi, J. Phys. A 41, 324002 (2008).

[2] A. J. Bray and M. A. Moore, in Heidelberg Colloquium on Glassy Dynamics, edited by J. L. van Hemmen and I. Morgenstern, Lecture Notes in Physics Vol. 275 (Springer, Berlin, 1986), pp. 121-153.

[3] D. S. Fisher and D. A. Huse, Phys. Rev. Lett. 56, 1601 (1986).

[4] M. Mézard, G. Parisi, and M. A. Virasoro, Spin-Glass Theory and Beyond, Lecture Notes in Physics Vol. 9 (World Scientific, Singapore, 1987).

[5] E. Marinari, G. Parisi, F. Ricci-Tersenghi, J. Ruiz-Lorenzo, and F. Zuliani, J. Stat. Phys. 98, 973 (2000).

[6] G. Parisi and F. Ricci-Tersenghi, J. Phys. A 33, 113 (2000).

[7] G. Parisi, PoS (HRMS) 023 (2010).

[8] S. Franz, G. Parisi, and M. A. Virasoro, J. Phys. I (France) 4, 1657 (1994).

[9] S. Boettcher, Phys. Rev. Lett. 95, 197205 (2005).

[10] C. de Dominicis, I. Kondor, and T. Temesvári, in Spin Glasses and Random Fields, edited by A. P. Young (World Scientific, Singapore, 1998).

[11] L. A. Fernández, V. Martin-Mayor, G. Parisi, and B. Seoane, Phys. Rev. B 81, 134403 (2010).

[12] G. Parisi, P. Ranieri, F. Ricci-Tersenghi, and J. J. Ruiz-Lorenzo, J. Phys. A 30, 7115 (1997).

[13] C. D. Dominicis and I. Giardina, Random Fields and Spin Glasses: A Field Theory Approach (Cambridge University, Cambridge, 2006).
[14] E. Marinari, G. Parisi, J. J. Ruiz-Lorenzo, and F. Ritort, Phys. Rev. Lett. 76, 843 (1996).

[15] E. Marinari, G. Parisi, F. Ricci-Tersenghi, and J. J. RuizLorenzo, J. Phys. A 33, 2373 (2000).

[16] F. Belletti et al., Phys. Rev. Lett. 101, 157201 (2008).

[17] F. Belletti et al., J. Stat. Phys. 135, 1121 (2009).

[18] E. Marinari and G. Parisi, Phys. Rev. B 62, 11677 (2000).

[19] E. Marinari and G. Parisi, Phys. Rev. Lett. 86, 3887 (2001).

[20] P. Contucci, C. Giardinà, C. Giberti, G. Parisi, and C. Vernia, Phys. Rev. Lett. 103, 017201 (2009).

[21] L. Berthier and J.-P. Bouchaud, Phys. Rev. B 66, 054404 (2002).

[22] T. Jörg and H. G. Katzgraber, Phys. Rev. B 77, 214426 (2008).

[23] Intel C++ Compiler User and Reference Guides, Intel document number 304968-023US (2010).

[24] C. Owens and R. Parikh, http://software.intel.com/en-us/ articles/fast-random-number-generator-on-the-intel-pentiumr4-processor/ (2009).

[25] J. Pommier, http://gruntthepeon.free.fr/ssemath/ (2007).

[26] D. Yllanes, arXiv:1111.0266.

[27] G. Parisi, F. Ricci-Tersenghi, and J. J. Ruiz-Lorenzo, J. Phys. A 29, 7943 (1996).

[28] W. H. Press, S. A. Teukolsky, W. T. Vetterling, and B. P. Flannery, Numerical Recipes (Cambridge University, Cambridge, 1992). 